\title{
Carbapenem-resistant Enterobacter cloacae complex in a tertiary Hospital in Northeast China, 2010-2019
}

Jingjing Chen ${ }^{1,2,3}$, Sufei Tian ${ }^{1,2,3}$, Hua Nian ${ }^{1,2,3}$, Ruixuan Wang ${ }^{1,2,3}$, Fushun $\mathrm{Li}^{1,2,3}$, Ning Jiang ${ }^{1,2,3}$ and Yunzhuo $\mathrm{Chu}^{1,2,3^{*}}$

\begin{abstract}
Background: Carbapenem-resistant Enterobacter cloacae complex (CREC) is a new emerging threat to global public health. The objective of the study was to investigate the clinical characteristics and molecular epidemiology of CREC infections in the medical center of northeast China.

Methods: Twenty-nine patients were infected/colonized with CREC during a ten-year period (2010-2019) by WHONET analysis. Antibiotic susceptibilities were tested with VITEK 2 and micro broth dilution method (for polymyxin B and tigecycline). Carbapenemase encoding genes, $\beta$-lactamase genes, and seven housekeeping genes for MLST were amplified and sequenced for 18 cryopreserved CREC isolates. Maximum likelihood phylogenetic tree was built with the concentrated sequences to show the relatedness between the 18 isolates.

Results: There was a rapid increase in CREC detection rate during the ten-year period, reaching $8.11 \%$ in 2018 and 6.48\% in 2019. The resistance rate of CREC isolates to imipenem and meropenem were 100.0 and $77.8 \%$, however, they showed high sensitivity to tigecycline, polymyxin B and amikacin. The 30-day crude mortality of CREC infection was $17.4 \%$, indicating that it may be a low-virulence bacterium. Furthermore, molecular epidemiology revealed that ST93 was the predominant sequence type followed by ST171 and ST145, with NDM-1 and NDM-5 as the main carbapenemase-encoding genes. Moreover, E. hormaechei subsp. steigerwaltii and E. hormaechei subsp. oharae were the main species, which showed different resistance patterns.

Conclusion: Rising detection rate of CREC was observed in a tertiary hospital, which showed heterogeneity in drug resistance patterns, resistance genes, and MLST types. Effective infection prevention and control measures should be taken to reduce the spread of CREC.
\end{abstract}

Keywords: Enterobacter cloacae complex, Carbapenem-resistant, Molecular analyses, ST93, NDM

\footnotetext{
* Correspondence: cyz6630@163.com

'Department of Laboratory Medicine, The First Affiliated Hospital of China

Medical University, 155 North Nanjing Street, Heping District, Shenyang

110001, Liaoning, China

${ }^{2}$ National Clinical Research Center for Laboratory Medicine, The First Affiliated

Hospital of China Medical University, Shenyang 110001, China

Full list of author information is available at the end of the article
}

(C) The Author(s). 2021 Open Access This article is licensed under a Creative Commons Attribution 4.0 International License, which permits use, sharing, adaptation, distribution and reproduction in any medium or format, as long as you give appropriate credit to the original author(s) and the source, provide a link to the Creative Commons licence, and indicate if changes were made. The images or other third party material in this article are included in the article's Creative Commons licence, unless indicated otherwise in a credit line to the material. If material is not included in the article's Creative Commons licence and your intended use is not permitted by statutory regulation or exceeds the permitted use, you will need to obtain permission directly from the copyright holder. To view a copy of this licence, visit http://creativecommons.org/licenses/by/4.0/. The Creative Commons Public Domain Dedication waiver (http://creativecommons.org/publicdomain/zero/1.0/) applies to the data made available in this article, unless otherwise stated in a credit line to the data. 


\section{Background}

Enterobacter cloacae complex (ECC) which comprises the following species, Enterobacter cloacae, Enterobacter hormaechei, Enterobacter asburiae, Enterobacter kobei, Enterobacter ludwigii, Enterobacter nimipressuralis, Enterobacter mori, etc., is an important Enterobacteriaceae widely encountered in the environment [1]. As an opportunistic pathogen, it has ranked as the top three Enterobacteriaceae in hospital-associated infections these years. ECC are found to be involved in multiple infections, such as bacteremia, respiratory tract infections, wound infections, urinary tract infections, nosocomial infections, etc. [2].

To date, carbapenem-resistant E. cloacae complex (CREC) has become as the third most common carbapenem-resistant Enterobacteriaceae (CRE) in China [3]. According to the surveillance of China Antimicrobial Surveillance network (CHINET), carbapenem resistance rates among ECC were $<1.0 \%$ in 2007. Surprisingly, it rapidly increased to about $10 \%$ in 2019. Carbapenems are regarded as a last choice for treating severe gramnegative bacterial infections. Although the consequences of CREC infections remain largely unknown, infections caused by CRE can lead to high mortality, long hospitalization and high hospitalization cost. Therefore, CREC may become a new emerging threat to public health [4].

Genes encoding carbapenemases (KPC, NDM, VIM, IMP, and OXA-48), which are usually present on the plasmids, are the main mechanism of carbapenem-resistance in CREC. Besides, overexpression of $\beta$-lactamases encoded by TEM, CTX-M, SHV, etc., membrane-associated mechanisms, such as porin defects (Omps, porins, and outer membrane permeability), and efflux pumps may also participate in carbapenem resistance [1]. Global surveillance showed diversification of sequence types and resistance genes in ECC. Besides, regional distribution of CREC is observed, with KPC predominant in North America, OXA-48 and VIM predominant in Europe, and NDM predominant in China [5].

Due to the unclear clinical characteristics and notable diversity of CREC, this study was therefore conducted to investigate the clinical characteristics and molecular epidemiology of CREC infection/colonization in the First Hospital of China Medical University, which is the medical center in northeast China. The present study will contribute to understanding this emerging carbapenemresistant pathogen, which are fundamental for further treatment, effective infection prevention and control.

\section{Materials and methods}

\section{Bacterial strains}

This study was performed in the First Affiliated Hospital of China Medical University, a tertiary teaching hospital with 2249 beds and also the medical center of northeast China. CREC was defined as E. cloacae complex strains resistant to imipenem or meropenem. Twenty-nine patients were infected/colonized with CREC from January 2010 to December 2019 through WHONET analysis. Determination of infection and colonization of the patients was performed by two clinicians. Among these, 18 isolates (one isolate per patient) were cryopreserved and further experiments were carried out. $16 \mathrm{~s}$ rRNA sequencing was performed to confirm the species, and $h s p 60$ typing was applied to discriminate the genetic clusters [6].

This is a retrospective study which was approved by the Medical Ethics Committees of the First Affiliated Hospital of China Medical University. The Medical Ethics Committees of the First Hospital of China Medical University waived the need of informed consent.

\section{Species identification and antimicrobial susceptibility testing}

The VITEK 2 system and the MALDI TOF MS (bioMérieux, France) were applied for isolate identification, and the VITEK 2 GN09 was used to test the antimicrobial susceptibilities of all isolates. Carbapenem resistance was verified by Etest or K-B diffusion method. Minimal inhibitory concentrations (MICs) of polymyxin B and tigecycline were determined by broth microdilution method for the 18 isolates. The resistance results of tigecycline were interpreted following the European Committee on Antimicrobial Susceptibility Testing (EUCAST) guidelines. Susceptibilities of the other drugs were determined by the criteria of Clinical and Laboratory Standards Institute (CLSI). All methods were carried out in accordance with relevant guidelines and regulations.

\section{Phenotypic screening for carbapenemases and sequencing of antimicrobial resistance genes}

The phenotypic detection of carbapenemases production was achieved by RAPIDEC CARBA NP (bioMérieux, France). Carbapenemase genes (KPC, NDM, IMP, VIM, and OXA48-like), $\beta$-lactamase genes (TEM, CTX-M, and $\mathrm{SHV}$ ) and MCR-1 were amplified by polymerase chain reaction (PCR) according to previous methods [7], then positive PCR products were subjected to commercial Sanger sequencing services (Beijing Genomics institution Co., Ltd., China). Sequences were analyzed by nucleotide homology comparison against GenBank database by BLAST.

\section{Multilocus sequence typing (MLST)}

The E. cloacae MLST was performed as described previously (https://pubmlst.org/ecloacae/) by sequencing seven housekeeping genes: dnaA, fusA, gyrB, leuS, pyrG, rplB, and rpoB [8]. Briefly, the genes were amplified and 
then DNA sequencing was performed for positive ones. Sequence types (ST) were assigned by uploading the sequences to the online Enterobacter cloacae typing database.

\section{Phylogenetic analysis}

The sequences were assembled with Contig software, edited with BioEdit and aligned with Clustal W tool present in BioEdit software. After combining the seven MLST genes and 16S rRNA gene together, maximum likelihood phylogenetic tree was constructed with MEGA 5.1 software to display the relatedness between the 18 isolates. Bootstrap analyses with 1000 replicates were applied.

\section{Statistical analyses}

Statistical analyses were performed with WHONET software 5.6 and SPSS 20.0 software. For all statistical analyses, $p$ value $<0.05$ was considered statistically significant.

\section{Results}

Basic characteristics of the CREC isolates

A total of 29 consecutive nonduplicate CREC isolates were identified during 2010-2019 (Table 1), which originated from different anatomical sites: urine $(n=8$, $27.6 \%)$, blood $(n=6,20.7 \%)$, drainage $(n=5,17.2 \%)$, sputum $(n=5,17.2 \%)$, puncture fluid $(n=1,3.4 \%)$, catheter $(n=1,3.4 \%)$, tissue $(n=1,3.4 \%)$, secretion $(n=1,3.4 \%)$, and semen $(n=1,3.4 \%)$. The majority of patients were

Table 1 Basic characteristics of included patients infected/colonized with CREC

\begin{tabular}{|c|c|c|c|c|c|c|c|}
\hline Isolation ID & Specimen & Infection/colinization & Patient age $^{\mathrm{a}}$ & Sex & Isolation date $^{\mathrm{b}}$ & Department & Outcome $^{c}$ \\
\hline CMU1 & blood & Infection & 51 & $\mathrm{~F}$ & $2012 / 4 / 18$ & Hematology ward & Survive \\
\hline CMU2 & secretion & Infection & 60 & M & 2014/9/17 & Otolaryngology clinic & Survive \\
\hline CMU3 & urine & Colonization & 77 & $\mathrm{~F}$ & $2015 / 4 / 27$ & Intensive care unit & Survive \\
\hline CMU4 & urine & Colonization & 57 & M & 2015/5/9 & Urinary surgery ward & Survive \\
\hline CMU5 & tissue & Infection & 29 & M & 2015/6/17 & Orthopedic ward & Survive \\
\hline CMU6 & urine & Infection & 60 & M & 2016/8/18 & Surgical clinic & Survive \\
\hline CMU7 & drainage & Infection & 52 & M & 2016/12/21 & Intensive care unit & Die \\
\hline CMU8 & urine & Infection & 86 & M & 2017/1/11 & Intensive care unit & Survive \\
\hline CMU9 & urine & Colonization & 11 & M & $2017 / 4 / 29$ & Infection ward & Survive \\
\hline CMU10 & sputum & Infection & 81 & $\mathrm{~F}$ & $2017 / 7 / 20$ & Intensive care unit & Survive \\
\hline CMU11 & drainage & Infection & 66 & M & $2017 / 8 / 4$ & Hepatobiliary surgery & Survive \\
\hline CMU12 & drainage & Infection & 54 & M & 2017/9/12 & Hepatobiliary surgery & Survive \\
\hline CMU13 & puncture fluid & Infection & 66 & M & 2017/11/7 & Emergency center & Survive \\
\hline CMU14 & urine & Infection & 72 & M & 2018/5/11 & Respiratory ward & Survive \\
\hline CMU15 & urine & Colonization & 61 & M & $2018 / 5 / 29$ & Urinary surgery ward & Survive \\
\hline CMU16 & semen & Infection & 54 & M & 2018/6/13 & Surgical clinic & Survive \\
\hline CMU17 & blood & Infection & 8 days & $\mathrm{F}$ & 2018/6/18 & Neonatal ward & Survive \\
\hline CMU18 & blood & Infection & 54 & M & 2018/7/12 & Respiratory ward & Survive \\
\hline CMU19 & blood & Infection & 68 & $\mathrm{~F}$ & 2018/7/19 & Neurosurgery ward & Survive \\
\hline CMU20 & sputum & Colonization & 82 & M & 2018/10/18 & Respiratory ward & Survive \\
\hline CMU21 & blood & Infection & 16 & M & 2018/11/22 & Intensive care unit & Survive \\
\hline CMU22 & sputum & Infection & 60 & M & 2018/12/19 & Intensive care unit & Die \\
\hline CMU23 & sputum & Infection & 77 & M & 2019/1/3 & Hepatobiliary surgery & Survive \\
\hline CMU24 & catheter & Infection & 52 & M & 2019/3/4 & Cardiac surgery ward & Die \\
\hline CMU25 & sputum & Colonization & 60 & M & 2019/3/22 & Cardiac surgery ward & Survive \\
\hline CMU26 & blood & Infection & 21 & M & 2019/4/11 & Intensive care unit & Survive \\
\hline CMU27 & drainage & Infection & 60 & $\mathrm{~F}$ & $2019 / 5 / 4$ & Intensive care unit & Die \\
\hline CMU28 & drainage & Infection & 33 & M & 2019/7/12 & Intensive care unit & Survive \\
\hline CMU29 & urine & Infection & 76 & M & 2019/11/17 & Urinary surgery ward & Survive \\
\hline
\end{tabular}

a years

${ }^{b}$ year/month/day

c survive or die within 30 days 
in the intensive care unit $(n=9,31.0 \%)$, followed by hepatobiliary surgical ward $(n=3,10.3 \%)$, urinary surgery ward $(n=3,10.3 \%)$, respiratory ward $(n=3,10.3 \%)$, cardiac surgery ward $(n=2,6.9 \%)$, surgical clinic $(n=2$, $6.9 \%)$, orthopedic ward $(n=1,3.4 \%)$, hematology ward $(n=1,3.4 \%)$, neurosurgery ward $(n=1,3.4 \%)$, neonatal ward $(n=1,3.4 \%)$, infection ward $(n=1,3.4 \%)$, otolaryngology clinic $(n=1,3.4 \%)$, and emergency center $(n=1$, $3.4 \%)$

The first isolate of CREC dates back to 2012 in the hematology ward. During the 10-year period, the CREC detection rate increased notably from $0.00 \%$ in 2010 to $6.48 \%$ in 2019 , with a peak of $8.11 \%$ in 2018 (Fig. 1). This rapid increase deserves further molecular epidemiology research.

\section{Clinical outcomes of CREC infections}

The overall 30-day crude mortality of CREC infection was $17.4 \%(4 / 23)$. The specimen types of the four patients were as follows: drainage $(n=2)$, catheter $(n=1)$, sputum $(n=1)$. None of the patients with bloodstream infections died. Furthermore, all four patients were accompanied with other infections: two patients with serious abdominal infection, one patient with cytomegalovirus pneumonia, and one patient with Acinetobacter baumannii bloodstream infection. This indicated that CREC may be not the main reason of death.

\section{Antimicrobial susceptibility testing}

The antimicrobial susceptibility testing was summarized in Table 2, which showed that $100.0 \%(18 / 18)$ isolates and $77.8 \%(14 / 18)$ isolates were resistant to imipenem and meropenem respectively. Among them, nine isolates showed extreme resistance ( $\mathrm{MIC} \geq 16$ ) to both drugs. In addition, these isolates showed high resistance to ceftriaxone (94.4\%), ceftazidime (94.4\%), piperacillin/tazobactam (77.8\%), cefepime (72.2\%), ciprofloxacin (66.7\%), and levofloxacin (61.1\%). Moreover, 55.6, 55.6, 44.4 and $44.4 \%$ strains were resistant to gentamicin, sulfamethoxazole-trimethoprim, nitrofurantoin and tobramycin respectively. In contrast, 100, 100 and 77.8\% of the isolates were susceptible to tigecycline, polymyxin $\mathrm{B}$ and amikacin.

\section{Resistance genes}

Carbapenemase producers were detected in 94.4\% (17/ 18 ) of the isolates (Table 3), and all the carbapenemaseproducing isolates harbored carbapenemase-encoding genes. Among them, four types of carbapenemases were detected in these isolates: blaNDM-1 $(n=9,50.0 \%)$, blaNDM-5 ( $n=7,38.9 \%)$, blaIMP-4 $(n=2,11.1 \%)$, and blaKPC-2 $(n=1,5.6 \%)$. Of note, co-occurrence of blaNDM-1 and blaIMP-4 was identified in two isolates (CMU10 and CMU29). For the $\beta$-lactamase genes, eight isolates had TEM-1, three isolates had CTX-M-15, three isolates had CTX-M-3, two isolates had SHV-12, and one isolate had CTX-M-14.

\section{MLST analysis}

It revealed 11 sequence types among the 18 CREC isolates, with ST93 as the predominant epidemic type $(n=$ $6,33.3 \%)$, followed by ST171 $(n=3,16.7 \%)$ and ST145 $(n=2,11.1 \%)$. The other types contained one isolate for each: ST13, ST66, ST114, ST528, ST1120 ( $n=1,5.6 \%)$.

\section{Clonal relatedness analysis}

To analyze the phylogenetic relationships between these 18 isolates, a maximum likelihood tree (Fig. 2A) was constructed with the concatenated sequences (6090 bp) of the seven loci MLST genes and $16 \mathrm{~s}$ rRNA, which

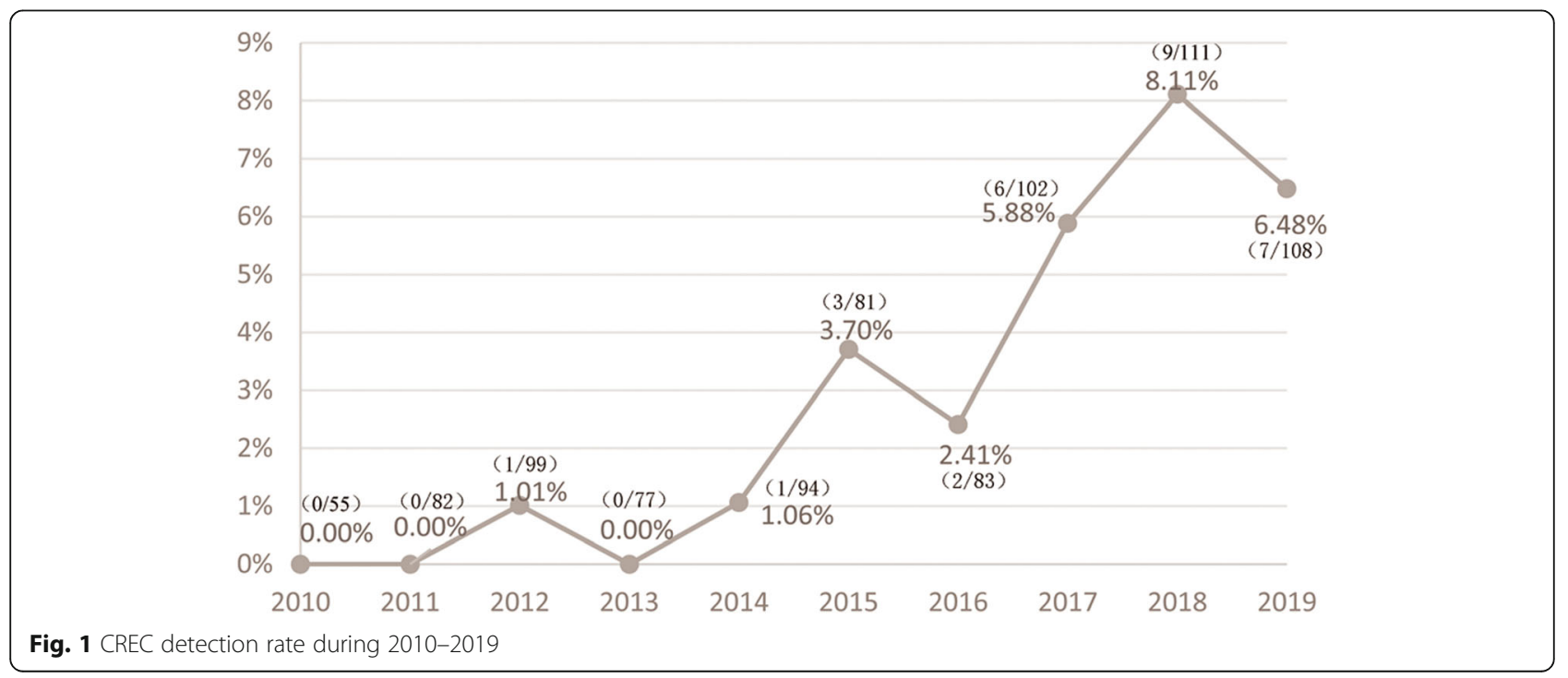


Table 2 Antimicrobial susceptibility results showing the MICs of 18 CREC isolates

\begin{tabular}{llllllllllllllllllll}
\hline $\begin{array}{ll}\text { Isolation } \\
\text { ID }\end{array}$ & CAZ & CRO & FEP & AMK & GEN & CIP & NIT & AZT & TOB & LEV & SXT & TZP & MEM & IMP & TGC & PB \\
\hline & & & & & & & & & & & & & $\leq 1 / 19$ & & & \\
CMU2 & $<=1$ & $<=1$ & $\leq 1$ & $\leq 2$ & $\leq 1$ & $\leq 0.25$ & 64 & $\leq 1$ & $\leq 1$ & $\leq 0.25$ & & 8 & 8 & $\geq 16$ & \\
CMU5 & $\geq 64$ & $\geq 64$ & $\geq 64$ & $\geq 64$ & $\geq 16$ & 1 & 64 & 16 & $\geq 16$ & 1 & $\geq 16 / 304$ & $\geq 128$ & 8 & $\geq 16$ & 0.5 & 1 \\
CMU6 & $\geq 64$ & $\geq 64$ & 32 & $\geq 64$ & $\geq 16$ & $\geq 4$ & 256 & $\geq 64$ & $\geq 16$ & $\geq 8$ & $\leq 1 / 19$ & $\geq 128$ & 2 & $\geq 16$ & 2 & 0.5 \\
CMU8 & $\geq 64$ & $\geq 64$ & 8 & 16 & 8 & 2 & 128 & $\geq 64$ & $\geq 16$ & 4 & $\leq 1 / 19$ & 64 & 1 & 4 & 2 & 1 \\
CMU10 & $\geq 64$ & $\geq 64$ & $\geq 64$ & $\leq 2$ & $\geq 16$ & $\leq 0.25$ & 32 & $\geq 64$ & 8 & 0.5 & $\geq 16 / 304$ & $\geq 128$ & $\geq 16$ & $\geq 16$ & 1 & 0.5 \\
CMU11 & $\geq 64$ & $\geq 64$ & 16 & $\leq 2$ & $\geq 16$ & $\leq 0.25$ & 32 & $\leq 1$ & $\geq 16$ & $\leq 0.25$ & $\leq 1 / 19$ & $\geq 128$ & 8 & $\geq 16$ & 0.25 & 1 \\
CMU12 & $\geq 64$ & $\geq 64$ & $\geq 64$ & $\leq 2$ & $\geq 16$ & 2 & 128 & $\geq 64$ & 8 & 2 & $\geq 16 / 304$ & $\geq 128$ & $\geq 16$ & $\geq 16$ & 0.25 & 2 \\
CMU13 & $\geq 64$ & $\geq 64$ & 4 & $\leq 2$ & $\leq 1$ & $\leq 0.25$ & 128 & $\geq 64$ & $\leq 1$ & $\leq 0.25$ & $\leq 1 / 19$ & 64 & $\geq 16$ & $\geq 16$ & 0.5 & 1 \\
CMU14 & $\geq 64$ & $\geq 64$ & $\geq 64$ & 8 & $\geq 16$ & $\geq 4$ & 64 & $\geq 64$ & $\geq 16$ & $\geq 8$ & $\geq 16 / 304$ & $\geq 128$ & 8 & 4 & 2 & 1 \\
CMU15 & $\geq 64$ & $\geq 64$ & 8 & $\leq 2$ & 8 & 0.5 & 64 & 2 & 8 & 1 & $\geq 16 / 304$ & 64 & 1 & 4 & 1 & 0.5 \\
CMU18 & $\geq 64$ & $\geq 64$ & $\geq 64$ & $\leq 2$ & $\leq 1$ & $\geq 4$ & 256 & 16 & $\leq 1$ & $\geq 8$ & $\geq 16 / 304$ & $\geq 128$ & $\geq 16$ & $\geq 16$ & 1 & 0.5 \\
CMU19 & $\geq 64$ & $\geq 64$ & 2 & 16 & 8 & $\leq 0.25$ & 64 & $\geq 64$ & $\geq 16$ & $\leq 0.25$ & $\leq 1 / 19$ & 32 & 1 & 8 & 0.5 & 1 \\
CMU23 & $\geq 64$ & $\geq 64$ & $\geq 64$ & $\leq 2$ & $\leq 1$ & $\geq 4$ & 32 & $\geq 64$ & $\leq 1$ & $\geq 8$ & $\geq 16 / 304$ & $\geq 128$ & $\geq 16$ & $\geq 16$ & 1 & 1 \\
CMU25 & $\geq 64$ & $\geq 64$ & $\geq 64$ & $\leq 2$ & $\geq 16$ & $\geq 4$ & 64 & $\geq 64$ & 8 & $\geq 8$ & $\geq 16 / 304$ & $\geq 128$ & 8 & $\geq 16$ & 1 & 1 \\
CMU26 & $\geq 64$ & $\geq 64$ & $\geq 64$ & $\geq 64$ & $\geq 16$ & $\geq 4$ & 256 & $\geq 64$ & $\geq 16$ & $\geq 8$ & $\leq 1 / 19$ & $\geq 128$ & $\geq 16$ & $\geq 16$ & 0.5 & 1 \\
CMU27 & $\geq 64$ & $\geq 64$ & $\geq 64$ & $\leq 2$ & $\leq 1$ & $\geq 4$ & 32 & $\geq 64$ & $\leq 1$ & $\geq 8$ & $\geq 16 / 304$ & $\geq 128$ & $\geq 16$ & $\geq 16$ & 1 & 0.5 \\
CMU28 & $\geq 64$ & $\geq 64$ & $\geq 64$ & $\geq 64$ & $\geq 16$ & $\geq 4$ & 256 & $\geq 64$ & $\geq 16$ & $\geq 8$ & $\leq 1 / 19$ & $\geq 128$ & $\geq 16$ & $\geq 16$ & 1 & 1 \\
CMU29 & $\geq 64$ & $\geq 64$ & $\geq 64$ & $\leq 2$ & $\geq 16$ & 1 & 128 & $\geq 64$ & 8 & 2 & $\geq 16 / 304$ & $\geq 128$ & $\geq 16$ & $\geq 16$ & 2 & 0.5 \\
R (\%) & 94.4 & 94.4 & 72.2 & 22.2 & 55.6 & 66.7 & 44.4 & 72.2 & 44.4 & 61.1 & 55.6 & 72.2 & 72.2 & 100.0 & 0.0 & 0.0 \\
\hline
\end{tabular}

Grey-shaded cells were interpreted as resistant

Abbreviations: CAZ ceftazidime, CRO ceftriaxone, FEP cefepime, AMK amikacin, GEN gentamicin, CIP ciprofloxacin, NIT nitrofurantoin, AZT aztreonam, TOB tobramycin, LEV levofloxacin, SXT sulfamethoxazole-trimethoprim, TZP piperacillin/tazobactam, MEM meropenem, IMP imipenem, TGC tigecycline, PB polymyxin B

Table 3 Carbapenemases production, resistance genes and sequence types of 18 CREC isolates

\begin{tabular}{|c|c|c|c|c|c|c|}
\hline Isolation ID & hsp60 typing & Craba NP & Carbapenemase-encoding genes & $\beta$-lactamase genes & MCR-1 & ST type \\
\hline CMU2 & E. hormaechei & + & NDM-5 & & - & ST250 \\
\hline CMU5 & E. kobei & + & NDM-5 & TEM-1, CTX-M-3 & - & ST145 \\
\hline CMU6 & E. hormaechei & + & $\mathrm{KPC}-2$ & TEM-1, CTX-M-3 & - & ST93 \\
\hline CMU8 & E. hormaecheic & + & NDM-1 & SHV-12 & - & ST66 \\
\hline CMU10 & E. hormaechei ${ }^{a}$ & + & NDM-1, IMP-4 & TEM-1 & - & ST93 \\
\hline CMU11 & E. hormaechei ${ }^{a}$ & + & NDM-1 & TEM-1 & - & ST93 \\
\hline CMU12 & E. hormaechei & + & NDM-1 & & - & ST93 \\
\hline CMU13 & E. ludwigii & - & & & - & ST13 \\
\hline CMU14 & E. hormaecheic & + & NDM-5 & CTX-M-15 & - & ST171 \\
\hline CMU15 & E. kobei & + & NDM-1 & & - & ST145 \\
\hline CMU18 & E. hormaecheic & + & NDM-1 & CTX-M-14 & - & ST114 \\
\hline CMU19 & E. hormaecheib & + & NDM-1 & SHV-12 & - & ST528 \\
\hline CMU23 & E. hormaechei & + & NDM-5 & CTX-M-15 & - & ST171 \\
\hline CMU25 & E. hormaechei & + & NDM-1 & TEM-1, CTX-M-3 & - & ST1120 \\
\hline CMU26 & E. hormaechei & + & NDM-5 & TEM-1 & - & ST93 \\
\hline CMU27 & E. hormaecheic & + & NDM-5 & CTX-M-15 & - & ST171 \\
\hline CMU28 & E. asburiae ${ }^{a}$ & + & NDM-5 & TEM-1 & - & ST93 \\
\hline CMU29 & E. hormaechei & + & NDM-1, IMP-4 & TEM-1 & - & ST93 \\
\hline
\end{tabular}

${ }^{\mathrm{a}}$ E. hormaechei subsp. steigerwaltii

${ }^{\mathrm{b}}$ E. hormaechei subsp. hormaechei

${ }^{C} E$. hormaechei subsp. oharae 


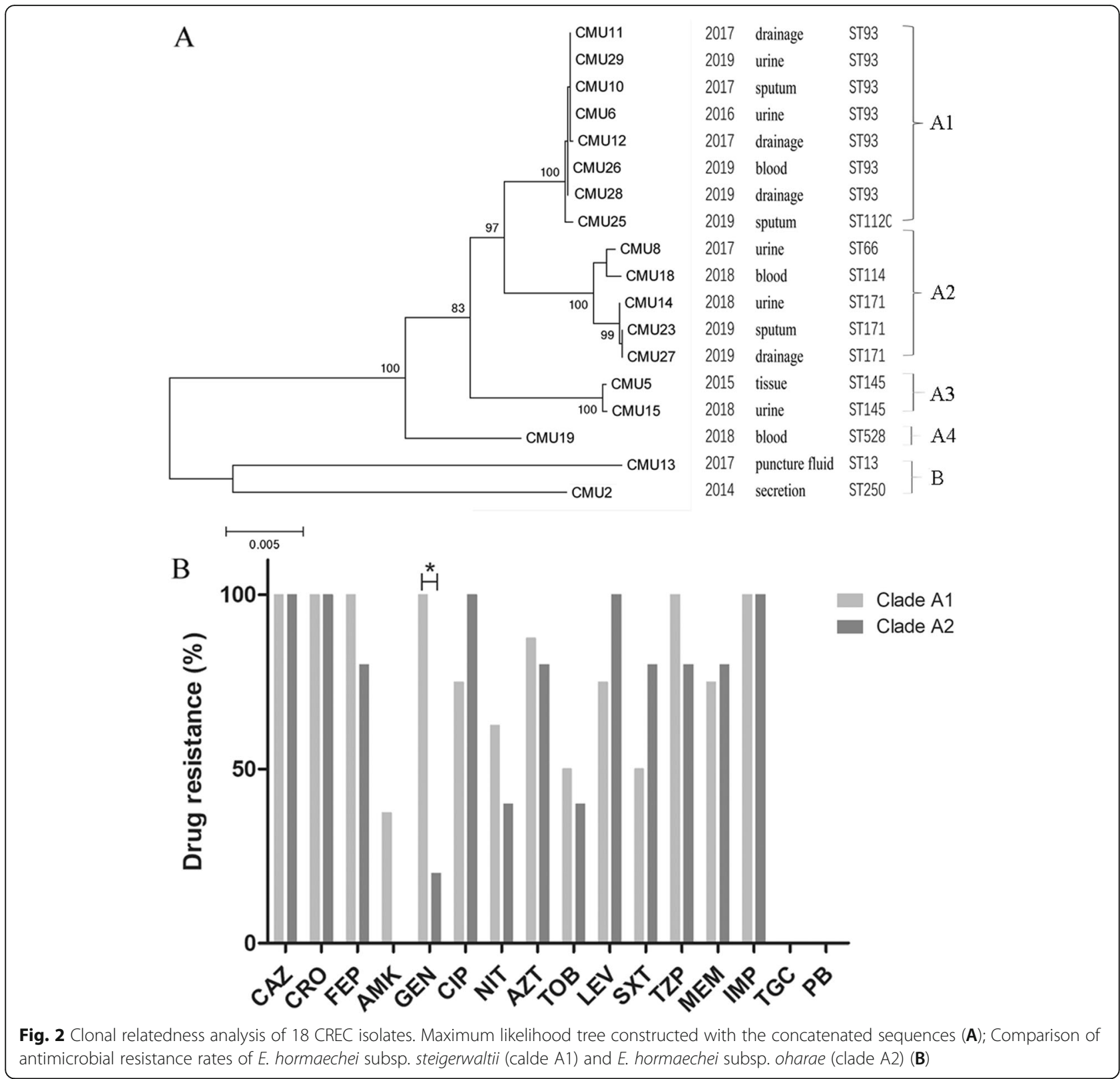

formed two separate clades. Clade A was sub-divided into four subclades, clade A1, A2, A3, A4. Clade A1 were E. hormaechei subsp. steigerwaltii typed as ST93 and ST 1120; Clade A2 were E. hormaechei subsp. oharae typed as ST66, ST114, and ST171; Clade A3 were E. kobei of ST145, Clade A4 were E. hormaechei subsp. hormaechei of ST528. Whereas clade B had two sequence types and were identified as $E$. ludwigii and $E$. asburiae respectively. In short, phylogenetic analysis of 18 CREC isolates showed genetic diversity with $E$. hormaechei as the predominant species.

Furthermore, we compared the antimicrobial resistance patterns of $E$. hormaechei subsp. steigerwaltii (clade A1) and E. hormaechei subsp. oharae (clade A2). As shown in Fig. 2B, clade A1 were characterized with higher gentamicin resistance rate relative to clade A2 $(p<0.05)$. However, due to the small sample size, further confirmation was needed.

\section{Discussion}

Wide spread of CREC poses a great threat to public health. Therefore, it is urgent to characterize the clinical molecular epidemiology of CREC infection in the medical center of northeast China. Results revealed that there was a rapid increase in CREC detection rate during 2010-2019, which showed high sensitivity to tigecycline, polymyxin $\mathrm{B}$ and amikacin through antimicrobial susceptibility test. The overall 30-day crude mortality of 
CREC infection was $17.4 \%$, indicating that CREC may be a low-virulence pathogen. Besides, molecular epidemiology indicated that ST93 was the predominant sequence type followed by ST171 and ST145, with NDM1 and NDM-5 as the main carbapenemase-encoding genes.

Since the first identification of CREC in our hospital in 2012, a rapid increase in the CREC detection rate was observed thereafter, reaching $8.11 \%$ in 2018 and $6.48 \%$ in 2019, indicating that CREC has become an escalating threat of nosocomial infection. This is consistent with previous surveillance of CHINET and the US Veterans Health Administration, which also reported an increase in the resistance rate of carbapenems in ECC $[9,10]$. Reasons explaining for the increase may be as follows: (1) Extensive use of broad-spectrum antibiotics (especially third or fourth generation cephalosporins and carbapenems), invasive devices (mechanical ventilation, central venous catheter, parenteral nutrition, urinary catheter, etc), surgical procedures, as well as prolonged hospitalization are associated with CREC development [11]. (2) ECC is characterized with remarkable ability to acquire resistance determinants, leading to a rapid increase of CREC [12].

In terms of the resistance profiles, 100.0 and $77.8 \%$ of the CREC isolates in our hospital were resistant to imipenem and meropenem respectively. CREC isolates showed heterogeneity in resistance patterns to imipenem and meropenem, with some isolates resistant to both imipenem and meropenem while other isolates resistant to imipenem and susceptible to meropenem. This indicates that multiple mechanisms may participate in carbapenem resistance of CREC isolates, such as production of carbapenemase; Besides, over-expression of multidrug efflux pumps and loss of outer membrane protein were reported to be associated with resistance to meropenem and imipenem respectively. For the imipenem-resistant and meropenem-susceptible isolates, NDM-1 carbapenemase and loss of outer membrane protein may be the reasons explaining this resistant phenotype, which needs to be verified in the future.

Moreover, CREC isolates showed medium sensitivity to gentamicin, sulfamethoxazole-trimethoprim, nitrofurantoin and tobramycin, and high sensitivity to tigecycline, polymyxin $\mathrm{B}$ and amikacin. These results demonstrated that there were limited treatment options for CREC, making it a threat of drug resistance. Combination of antibiotics, such as meropenem, polymyxin $B$, tigecycline, and amikacin showed promising synergy results $[13,14]$. However, optimal treatment combinations for different sequence types and resistant genotypes should be further evaluated. In addition, isolate CMU2 containing NDM-5 gene alone showed highly susceptibility to third and fourth generation cephalosporins as well as other antibiotics except meropenem and imipenem, which is unusual for carbapenemase producing organisms. Further investigation into the underlying mechanism is warranted.

Furthermore, our study revealed that the overall 30day crude mortality of CREC infection was $17.4 \%$. Previous meta-analysis showed that pooled crude mortality of carbapenem resistant Klebsiella pneumoniae was 42.1\% [15]. Other studies reported the 30-day mortality of carbapenem-resistant Pseudomonas aeruginosa was $36.6 \%$ [16], whereas the mortality of carbapenemresistant A. baumannii ranged from 16 to $76 \%$ [17], which were higher than the crude mortality of CREC in our hospital. Besides, all four patients who died were accompanied with other serious infections. Taken together, these indicated that CREC may be a low-virulence pathogen which deserves further validation.

Molecular epidemiology analyses were undertaken for the 18 CREC strains from our hospital. MLST analysis revealed diverse sequence types with ST93 as the predominant type followed by ST171 and ST145. ST93 was frequently reported in China, such as Hangzhou, Nanjing, Jiamusi, etc. [18-20], whereas ST171 was commonly reported the U.S.A. and Japan [21, 22]. The diversification of the sequence types of CREC in our hospital is consistent with previous studies which also showed genetic heterogeneity [10]. For the resistance genes, NDM-1 and NDM-5 were the predominant carbapenemase-encoding genes, and TEM- 1 was the most common $\beta$-lactamase gene.

CREC is an emerging multi-drug resistant pathogen, which are associated with the risk of spreading to the communities. Therefore, it is imperative to take effective infection prevention and control practices to confront this threat [21]. However, the wide genotypic diversity of CREC isolates may indicate that CREC has strong ability to acquire drug resistance genes, thereby increasing the difficulty in infection prevention and control. Above all, screening of carbapenem resistant pathogens should be conducted, especially in patients with high risks. Moreover, other infection and control measures, including rational use of antibiotics, environment cleaning, faecal and medical waste management, hand hygiene, staff education, etc. should be implemented to curb the global spread of CREC [23].

The current study has some limitations. Firstly, the number of isolates is relatively small because CREC were infrequent despite the increasing detection rate in our hospital. Secondly, this was a retrospective study in a single center, and 11 out of 29 CREC isolates were not cryopreserved, which may lead to bias. Thirdly, although the mortality of CREC is low compared with other carbapenem resistant pathogens, which indicates its low virulence, further virulence-based experiments should be performed. 
In conclusion, a rapid increase of CREC was observed during 2010-2019 in our hospital, which were with limited treatment options. Molecular epidemiology demonstrated the diversification of CREC, with ST93 as the predominant sequence type and NDM as the main carbapenemase-encoding gene. Intensive surveillance and effective measures should be undertaken to reduce the spread and transmission of CREC in the hospitals.

\section{Abbreviations}

CREC: Carbapenem-resistant Enterobacter cloacae complex; ECC: Enterobacter cloacae complex; CRE: Carbapenem-resistant Enterobacteriaceae; CHIN ET: China Antimicrobial Surveillance network; MICs: Minimal inhibitory concentrations; EUCAST: European Committee on Antimicrobial Susceptibility Testing; CLSI: Clinical and Laboratory Standards Institute; PCR: Polymerase chain reaction; MLST: multilocus sequence typing; ST: Sequence type

\section{Supplementary Information}

The online version contains supplementary material available at https://doi. org/10.1186/s12879-021-06250-0.

Additional file 1: Table S1. Primers for polymerase chain reactions in this present study. Table S2. MLST analysis of 18 CREC isolates.

\section{Acknowledgments}

We thank Dr. Xiaoli Zhang from Yongchuan hospital of Chongqing Medical University for her kind assistance.

\section{Authors' contributions}

CY and CJ conceived and designed the study; CJ, TS, WR and LF collected the data; $\mathrm{NH}, \mathrm{JN}$ and $\mathrm{CJ}$ analyzed the data; $\mathrm{CJ}$ and $\mathrm{CY}$ led the writing. All authors read and approved the final manuscript.

\section{Funding}

This work was supported by the CAMS Innovation Fund for Medical Sciences (2019-I2M-5-027).

\section{Availability of data and materials}

All data generated or analyzed during this study were included in this article and the supplementary information files. The phylogenetic datasets analyzed during the current study are available in the TreeBASE repository (http://purl. org/phylo/treebase/phylows/study/TB2:S28058, accession number: 28058).

\section{Declarations}

\section{Ethics approval and consent to participate}

This study was approved by the Medical Ethics Committees of the First Hospital of China Medical University. The Medical Ethics Committees of the First Hospital of China Medical University waived the need of informed consent.

\section{Consent for publication}

Not applicable.

\section{Competing interests}

No competing interests relevant to this article exist.

\section{Author details}

${ }^{1}$ Department of Laboratory Medicine, The First Affiliated Hospital of China Medical University, 155 North Nanjing Street, Heping District, Shenyang 110001, Liaoning, China. ${ }^{2}$ National Clinical Research Center for Laboratory Medicine, The First Affiliated Hospital of China Medical University, Shenyang 110001, China. ${ }^{3}$ Labortory Medicine Innovation Unit, Chinese Academy of Medical Sciences, Shenyang, China.
Received: 18 March 2021 Accepted: 27 May 2021

Published online: 26 June 2021

\section{References}

1. Davin-Regli A, Lavigne JP, Pages JM. Enterobacter spp.: update on taxonomy, clinical aspects, and emerging antimicrobial resistance. Clin Microbiol Rev. 2019;32(4):e00002-19. https://doi.org/10.1128/CMR.00002-19.

2. Mezzatesta ML, Gona F, Stefani S. Enterobacter cloacae complex: clinical impact and emerging antibiotic resistance. Future Microbiol. 2012;7(7):887902.https://doi.org/10.2217/fmb.12.61

3. Zhang Y, Wang Q, Yin Y, Chen H, Jin L, Gu B, et al. Epidemiology of Carbapenem-resistant Enterobacteriaceae infections: report from the China CRE network. Antimicrob Agents Chemother. 2018;62(2):e1882-17. https:// doi.org/10.1128/AAC.01882-17.

4. Davin-Regli A, Pages JM. Enterobacter aerogenes and Enterobacter cloacae; versatile bacterial pathogens confronting antibiotic treatment. Front Microbiol. 2015;6:392. https://doi.org/10.3389/fmicb.2015.00392.

5. Annavajhala MK, Gomez-Simmonds A, Uhlemann AC. Multidrug-resistant Enterobacter cloacae complex emerging as a global, diversifying threat. Front Microbiol. 2019;10:44. https://doi.org/10.3389/fmicb.2019.00044.

6. Hoffmann H, Roggenkamp A. Population genetics of the nomenspecies Enterobacter cloacae. Appl Environ Microbiol. 2003;69(9):5306-18. https:// doi.org/10.1128/aem.69.9.5306-5318.2003.

7. Poirel L, Walsh TR, Cuvillier V, Nordmann P. Multiplex PCR for detection of acquired carbapenemase genes. Diagn Microbiol Infect Dis. 2011;70(1):11923. https://doi.org/10.1016/j.diagmicrobio.2010.12.002.

8. Miyoshi-Akiyama T, Hayakawa K, Ohmagari N, Shimojima M, Kirikae T. Multilocus sequence typing (MLST) for characterization of Enterobacter cloacae. PLoS One. 2013;8(6):e66358. https://doi.org/10.1371/journal.pone. 0066358.

9. Wilson BM, El Chakhtoura NG, Patel S, Saade E, Donskey CJ, Bonomo RA, et al. Carbapenem-resistant Enterobacter cloacae in patients from the US veterans health administration, 2006-2015. Emerg Infect Dis. 2017;23(5):87880. https://doi.org/10.3201/eid2305.162034.

10. Gomez-Simmonds A, Hu Y, Sullivan SB, Wang Z, Whittier S, Uhlemann AC. Evidence from a new York City hospital of rising incidence of genetically diverse carbapenem-resistant Enterobacter cloacae and dominance of ST171, 2007-14. J Antimicrob Chemother. 2016;71(8):2351-3. https://doi. org/10.1093/jac/dkw132.

11. Tian X, Huang C, Ye X, Jiang H, Zhang R, Hu X, et al. Carbapenem-resistant Enterobacter cloacae causing nosocomial infections in southwestern China: molecular epidemiology, risk factors, and predictors of mortality. Infect Drug Resist. 2020;13:129-37. https://doi.org/10.2147/IDR.S234678.

12. Gomez-Simmonds A, Annavajhala MK, Wang Z, Macesic N, Hu Y, Giddins $\mathrm{MJ}$, et al. Genomic and geographic context for the evolution of high-risk carbapenem-resistant Enterobacter cloacae complex clones ST171 and ST78. mBio. 2018;9(3):e00542-18. https://doi.org/10.1128/mBio.00542-18.

13. Zhao Y, Li C, Zhang J, Fu Y, Hu K, Su S, et al. The in vitro activity of polymyxin $B$ and tigecycline alone and combination with other antibiotics against carbapenem-resistant Enterobacter cloacae complex isolates, including high-risk clones. Ann Transl Med. 2019;7(23):779. https://doi.org/1 0.21037/atm.2019.11.33.

14. Alves PH, Boff RT, Barth AL, Martins AF. Synergy of polymyxin B, tigecycline and meropenem against carbapenem-resistant Enterobacter cloacae complex isolates. Diagn Microbiol Infect Dis. 2019;94(1):81-5. https://doi. org/10.1016/j.diagmicrobio.2018.11.011.

15. Xu L, Sun X, Ma X. Systematic review and meta-analysis of mortality of patients infected with carbapenem-resistant Klebsiella pneumoniae. Ann Clin Microbiol Antimicrob. 2017;16(1):18. https://doi.org/10.1186/s12941-017-0191-3.

16. Urzedo JE, de Paula Menezes R, Porto JP, Ferreira ML, Goncalves IR, de Brito CS, et al. High mortality by nosocomial infections caused by carbapenemresistant $P$. aeruginosa in a referral hospital in Brazil: facing the perfect storm. J Med Microbiol. 2020;69(12):1388-97. https://doi.org/10.1099/jmm.0. 001273

17. Lemos EV, de la Hoz FP, Einarson TR, McGhan WF, Quevedo E, Castaneda C, et al. Carbapenem resistance and mortality in patients with Acinetobacter baumannii infection: systematic review and meta-analysis. Clin Microbiol Infect. 2014;20(5):416-23. https://doi.org/10.1111/1469-0691.12363.

18. Sun $L, X u J$, He F. Draft genome sequence data of a tigecycline-resistant Enterobacter cloacae ST93 clinical strain isolated from bloodstream infection. Data Brief. 2018;21:414-8. https://doi.org/10.1016/j.dib.2018.10.004. 
19. Cao XL, Cheng L, Zhang ZF, Ning MZ, Zhou WQ, Zhang K, et al. Survey of clinical extended-Spectrum Beta-lactamase-producing Enterobacter cloacae isolates in a Chinese tertiary hospital, 2012-2014. Microb Drug Resist. 2017; 23(1):83-9. https://doi.org/10.1089/mdr.2015.0128.

20. Zhao Y, Zhang J, Fu Y, Li C, Hu K, Su S, et al. Molecular characterization of metallo-beta-lactamase- producing carbapenem-resistant Enterobacter cloacae complex isolated in Heilongjiang Province of China. BMC Infect Dis. 2020;20(1):94. https://doi.org/10.1186/s12879-020-4768-7.

21. Pereira EC, Anacker M, Houseman J, Horn ME, Johnson TJ, Lynfield R, et al. A cluster of carbapenemase-producing Enterobacter cloacae complex ST171 at a tertiary care center demonstrating an ongoing regional threat. Am J Infect Control. 2019;47(7):767-72. https://doi.org/10.1016/j.ajic.2018.12.016.

22. Harada K, Shimizu T, Mukai Y, Kuwajima K, Sato T, Kajino A, et al. Phenotypic and molecular characterization of antimicrobial resistance in Enterobacter spp. isolates from companion animals in Japan. PLoS One. 2017:12(3): e0174178. https://doi.org/10.1371/journal.pone.0174178.

23. Magiorakos AP, Burns K, Rodriguez Bano J, Borg M, Daikos G, Dumpis U, et al. Infection prevention and control measures and tools for the prevention of entry of carbapenem-resistant Enterobacteriaceae into healthcare settings: guidance from the European Centre for Disease Prevention and Control. Antimicrob Resist Infect Control. 2017;6(1):113. https://doi.org/10.1186/s13756-017-0259-z.

\section{Publisher's Note}

Springer Nature remains neutral with regard to jurisdictional claims in published maps and institutional affiliations.

Ready to submit your research? Choose BMC and benefit from:

- fast, convenient online submission

- thorough peer review by experienced researchers in your field

- rapid publication on acceptance

- support for research data, including large and complex data types

- gold Open Access which fosters wider collaboration and increased citations

- maximum visibility for your research: over $100 \mathrm{M}$ website views per year

At $\mathrm{BMC}$, research is always in progress.

Learn more biomedcentral.com/submissions 